\title{
取向碳纳米管/高分子新型复合材料的制备及应用
}

\author{
丘龙斌孙雪梅仰志斌郭文瀚彭慧胜* \\ (复旦大学聚合物分子工程国家重点实验室 高分子科学系 先进材料实验室 上海 200438)
}

\begin{abstract}
摘要 碳纳米管/高分子复合材料已经被广泛研究，但长期以来存在一个共同而关键的挑战，即碳纳米管无规聚集，结 构难以调控, 性能无法满足应用需要. 本工作提出了制备取向碳纳米管/高分子复合材料的一种新方法, 获得块状、膜 状、纤维状复合材料, 制备的关键步骤是通过化学气相沉积法合成可纺的高质量碳纳米管阵列. 该方法简单易行，具有 较好的普适性. 由于碳纳米管取向排列, 复合材料具有优异的物理性能, 如碳纳米管取向后复合材料的机械强度和导 电率可分别提高一个和三个数量级. 在此基础上, 进一步探讨取向碳纳米管/高分子复合材料作为新型电极在有机太阳 能电池中的应用.
\end{abstract}

关键词 取向; 碳纳米管; 高分子; 复合材料

\section{Preparation and Application of Aligned Carbon Nanotube/Polymer Composite Material}

\author{
Qiu, Longbin $\quad$ Sun, Xuemei Yang, Zhibin Guo, Wenhan $\quad$ Peng, Huisheng* \\ (State Key Laboratory of Molecular Engineering of Polymers, Department of Macromolecular Science, and Laboratory of \\ Advanced Materials, Fudan University, Shanghai 200438)
}

\begin{abstract}
Carbon nanotube (CNT)/polymer composite materials have been widely studied for two decades. However, there remains a common and critical challenge, i.e., random dispersion of CNTs in polymer matrices, which has largely lowered their properties and limited their applications. Herein, we have developed a general method to prepare highly aligned $\mathrm{CNT}$ /polymer composite materials in formats of array, film, and fiber. The key procedure is to synthesize spinnable CNT arrays with high quality by a chemical vapor deposition process. $\mathrm{Fe} / \mathrm{Al}_{2} \mathrm{O}_{3}$ was used as catalyst, ethylene was used as carbon source, a mixture gas of argon and hydrogen was used as carrying gas. The optimal growth conditions were summarized as below: thickness of $1.2 \mathrm{~nm}$ for $\mathrm{Fe}$, thickness of $3 \mathrm{~nm}$ for $\mathrm{Al}_{2} \mathrm{O}_{3}$, flow rate of 400 standard $\mathrm{cm}^{3} / \mathrm{min}$ for argon, flow rate of 90 standard $\mathrm{cm}^{3} / \mathrm{min}$ for ethylene, flow rate of 30 standard $\mathrm{cm}^{3} / \mathrm{min}$ for hydrogen, growth temperature of $740{ }^{\circ} \mathrm{C}$, and growth time of $10 \mathrm{~min}$. Here the catalyst system was coated on silicon substrate by electron beam evaporation with rates of 0.5 and 2 $\AA / \mathrm{s}$ for Fe and $\mathrm{Al}_{2} \mathrm{O}_{3}$, respectively. To prepare CNT sheets or fibers, the spinnable array was first stabilized in a stage. A blade was then used to draw a ribbon out of the array. A CNT sheet would be obtained if the ribbon was directly pulled out without rotation, while a fiber should be produced if a rotary spinning was used. The spinning speed was about $15 \mathrm{~cm} / \mathrm{min}$. Monomer/polymer solutions or melts were directly coated onto the aligned CNT sheet or fiber to produce the aligned CNT/polymer film or fiber. Due to the high alignment of CNTs in polymer matrices, the resulting composite materials exhibited remarkable physical properties, e.g., the mechanical strength and electrical conductivity can be improved for one and three orders compared with the conventional solution blending method, respectively. These novel composite materials are promising for a wide variety of applications. The use of them as novel counter electrodes to fabricate dye-sensitized solar cells has been investigated as a demonstration. In a typical fabrication, an aligned CNT/polymer film (typical thickness of $5 \mu \mathrm{m}$ ) was first transferred onto fluorine doped tin oxide as counter electrode. A layer of $\mathrm{TiO}_{2}$ was coated onto fluorine doped tin oxide as working electrode, followed by immersion into $0.5 \mathrm{mmol} / \mathrm{L}$ cis-diisothiocyanato-bis(2,2'-bipyridyl-4,4'-dicarboxylato) ruthenium(II) bis(tetrabutylammonium) (also called N719) solution in a mixture solvent of acetonitrile/tert-butanol (volume ratio of 1/1) for $16 \mathrm{~h}$. After being further rinsed with acetonitrile and dried, the $\mathrm{N} 719$-incorporated $\mathrm{TiO}_{2}$ electrode was assembled with the counter electrode. An electrolyte which consisted of LiI, $\mathrm{I}_{2}, 2-2-3$-with propyl methyl 5-membered imidazole iodine, GuSCN, and tri-butyl-phosphate in dehydrated acetonitrile was injected into the cell through a hole on the counter electrode. The hole was finally sealed with surlyn and a piece of glass.

Keywords aligned; carbon nanotube; polymer; composite material
\end{abstract}

\footnotetext{
*E-mail: penghs@fudan.edu.cn

Received March 20, 2012; published April 17, 2012.
}

Supporting information for this article is available free of charge via the Internet at http://sioc-journal.cn

Project supported by the National Natural Science Foundation of China (Nos. 20904006, 91027025), Ministry of Science and Technology (Nos. 2011CB932503, 2011DFA51330), Ministry of Education (No. NCET-09-0318), Science and Technology Commission of Shanghai Municipality (Nos. 1052nm01600, 11520701400).

项目受国家自然科学基金(Nos. 20904006，91027025)、科技部(Nos. 2011CB932503，2011DFA51330)、教育部(NCET-09-0318)以及上海市科委(Nos. $1052 \mathrm{~nm} 01600,11520701400)$ 资助. 


\section{1 引言}

碳纳米管是由石墨片层卷绕而成的中空管. 根据石 墨片层的数目, 可以把碳纳米管分为单壁碳纳米管和多 壁碳纳米管 ${ }^{[1]}$. 这些独特的结构赋予了碳纳米管优异的 力学、电学性能, 如杨氏模量高达 $1000 \mathrm{GPa}$ (5 倍于钢), 拉伸强度高达 $63 \mathrm{GPa}$ (大约是钢的 50 倍) ${ }^{[2]}$. 由于碳纳米 管较低的密度(约为钢的 $1 / 6$ )、较高的强度、较高的电导 率以及其他优异的物理性能, 碳纳米管被认为在结构材 料、电子器件、场发射、生物医药和电化学等广泛领域 有着巨大的应用前景 ${ }^{[3 \sim 10]}$.

自 1991 年 Iijima $^{[11]}$ 发现碳纳米管以来, 碳纳米管吸 引了学术界与工业界广泛的兴趣, 但是碳纳米管的真正 应用仍然有限, 这是因为单根碳纳米管还存在一些亟需 解决的问题, 如分离成本高、结构难以精确控制和重复 等, 难以大规模应用到各种纳米器件和材料中 ${ }^{[12]}$. 一个 有效的解决途径是制备碳纳米管复合材料, 特别是与高 分子复合形成宏观尺度的功能材料, 这样碳纳米管间微 小的结构差异几乎不会对复合材料性能产生影响, 因此 具有较好的重复性并且可以大规模制备 ${ }^{[13]}$. 在碳纳米 管/高分子复合材料中, 碳纳米管赋予材料高强度、高电 导率、高热稳定性等优异的物理性能, 而高分子赋予材 料柔性、低成本、易加工等优异的性能. 事实上, 这些 复合材料已经开始应用在人们的生活中. 目前使用比较 广泛的是在塑料中引入碳纳米管制备抗静电的复合材 料. 一些高端体育产品如网球拍引入碳纳米管复合材 料, 提高产品的机械性能. 在 21 世纪, 碳纳米管/高分子 复合材料两个主要应用方向是高性能结构材料和能源 领域 ${ }^{[12]}$.

目前制备碳纳米管/高分子复合材料主要有三种方 法: 溶液混合、熔融混合 ${ }^{[13]}$ 和原位合成 ${ }^{[14]}$. 虽然这些方 法已经被广泛研究 ${ }^{[15 ~ 20]}$, 但它们面临一个共同而关键 的挑战: 碳纳米管在高分子中无规聚集, 复合材料无法 充分利用碳纳米管优异的性能, 如拉伸强度和电导率分 别低于 $0.12 \mathrm{GPa}$ 和 $10^{-6} \mathrm{~S} / \mathrm{cm}^{[13]}$, 严重限制碳纳米管/高 分子复合材料在很多领域的应用. 为此, 国际上多个课 题组做了大量研究工作, 包括尝试了外加电场 ${ }^{[21]}$ 、机械 拉伸 ${ }^{[22]} 、$ 旋转浇铸 ${ }^{[23]}$ 、熔融纤维纺丝 ${ }^{[24]}$ 、和静电纺丝技 术 ${ }^{[25 ~ 27]}$ 等多种方法, 试图解决碳纳米管无规聚集的难 题, 提高碳纳米管的取向性. 但是, 碳纳米管的取向度 和结构可控性依然很低, 复合材料的性能亟需进一步提 高.

近年来, 国际上多个课题组分别报道了碳纳米管阵 列、栅和纤维材料 ${ }^{[28}$-39], 其中碳纳米管栅和纤维通过干 法纺丝从可纺的碳纳米管阵列中获得. 在这些材料中碳 纳米管高度取向排列, 并且其结构和密度可在一定范围 内进行调控. 以这些取向碳纳米管材料作为模板, 然后 通过溶液或熔融方法引入高分子，可制备取向碳纳米管/ 高分子复合材料. 在这个方法中, 关键是合成高质量和
具有可纺性的碳纳米管阵列. 关于可纺阵列的合成已经

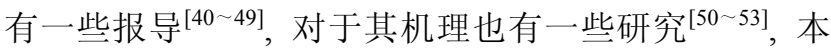
论文比较系统研究了相关实验参数对阵列可纺性的影 响规律，从而制备高质量的可纺阵列和碳纳米管栅与纤 维, 在此基础上获得具有优异机械和电学性能的取向碳 纳米管/高分子复合材料，并进一步探讨其作为新型电 极在有机太阳能电池中的应用.

\section{2 结果与讨论}

\section{1 可纺碳纳米管阵列的制备}

本文通过常压化学气相沉积法制备碳纳米管阵列, 一般碳纳米管阵列不具有可纺性, 这里可纺性指碳纳米 管可以从阵列中连续地拉出, 长度至少超过 $1 \mathrm{~m}$. 为了 制备可纺的碳纳米管阵列, 一般需要满足以下三个要 求，即窄的碳纳米管管径分布、合适的碳纳米管成核密 度、干净的碳纳米管表面 ${ }^{[54]}$. 这些参数可以通过改变催 化剂和生长条件来调节, 如在生长过程中控制催化剂粒 子的粒径分布密度, 可以满足前面两个要求, 而减少生 长过程中无定形碳的生成可以制备干净的碳纳米管.下 面对影响阵列可纺性的主要因素进行系统的研究.

\section{1 .1 催化剂的制备}

对于通过化学气相沉积法制备碳纳米管阵列, 常用 的催化剂包括铁、钴等金属, 催化剂可以通过物理蒸镀 或化学溶液方法沉积在基底上(通常为硅片). 为了延长 催化剂的活性，必须进一步在基底和催化剂之间引入三 氧化二铝缓冲层, 主要防止在高温时催化剂纳米粒子与 基底发生相互作用, 导致催化剂纳米粒子失活 ${ }^{[42]}$. 本论 文主要研究铁催化剂, 铁和三氧化二铝均通过电子束蒸 发来制备, 使用的基底为表面处理的硅片, 即硅片表面 有一层 $1 \mu \mathrm{m}$ 厚的二氧化硅.

(1)蒸镀时腔体压力的影响

分别使用了 $6.5 \times 10^{-5}, 1.0 \times 10^{-4}, 3 \times 10^{-4}, 5 \times 10^{-4}$, $7 \times 10^{-4}, 1 \times 10^{-3} \mathrm{~Pa}$ 等不同初始腔体压力蒸镀催化剂. 实验发现，当绝对压力大于或等于 $5 \times 10^{-4} \mathrm{~Pa}$ 时, 制备 的催化剂可以生长出可纺的阵列. 目前对于腔体绝对压 力的影响机理尚不明确, 可能腔体中含有少量氧气更加 有利于高质量催化剂的制备, 需要进一步深入研究. 另 外我们发现, 绝对压力越小, 制备的催化剂越有利于生 长出高的碳纳米管阵列.

(2)蒸镀速率的影响

一般认为沉积速率越慢, 得到的催化剂膜越均匀 ${ }^{[50]}$, 越适合制备碳纳米管阵列，但我们的实验发现，当蒸镀 速率过低时, 得到的催化剂不能生长出阵列; 而蒸镀速 率过高时, 虽然可以生长出阵列, 但阵列不可纺. 蒸镀 速率主要影响催化剂表面形貌、粗糙度以及沉积膜的密 度与规整度, 从而影响生长过程中催化剂膜裂解成颗粒 的过程. 因此存在最合适的蒸镀速率, 制备满足可纺阵 列要求的催化剂. 
在本体系中，当三氧化二铝的沉积速率在 $0.4 \sim 0.5$ $\AA / \mathrm{s}$, 铁的沉积速率在 $0.2 \AA / \mathrm{s}$ 时, 得到的催化剂不能制 备阵列. 当蒸镀速率进一步增加后, 得到的催化剂可用 于制备可纺的阵列，三氧化二铝最佳的蒸镀速率为 2.0 $\AA / \mathrm{s}$, 铁最佳的蒸镀速率为 $0.5 \AA / \mathrm{s}$.

(3)催化剂厚度的影响

催化剂厚度对于碳纳米管阵列的可纺性具有重要

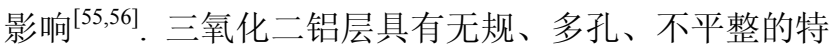
点, 有利于上层铁膜裂解成颗粒, 并可能影响铁颗粒的 大小. 铁膜的厚度直接影响了铁颗粒的大小及密度. 薄 膜产生相对较小但密度较高的铁颗粒, 厚膜产生得到较 大而密度较小的铁颗粒. 但当铁膜过薄时, 形成的铁颗 粒密度也会降低而无法形成阵列. 当铁膜过厚时, 铁颗 粒进一步聚集成大颗粒, 得到的阵列不可纺. 下面举例 说明. 固定三氧化二铝缓冲层厚度为 $5 \mathrm{~nm}$ 或 $20 \mathrm{~nm}$, 然 后改变铁膜厚度, 在 $0.8 \sim 2.0 \mathrm{~nm}$ 范围内每隔 $0.2 \mathrm{~nm}$ 取 一个点, 制备催化剂并生长碳纳米管阵列. 结果表明, 铁膜厚度在 $0.8 \sim 1.4 \mathrm{~nm}$ 范围内, 得到的阵列都具有一 定的可纺性, 但只有当铁膜厚度为 $1.2 \mathrm{~nm}$ 时能连续纺出 长度超过 $1 \mathrm{~m}$ 的碳纳米管栅或纤维, 其他厚度铁膜生长 的碳纳米管阵列只能纺出长度小于 $1 \mathrm{~m}$ 的栅或纤维.

当固定铁膜厚度为 $1.2 \mathrm{~nm}$ 后, 改变三氧化二铝缓冲 层厚度分别为 $1,2,3,4,5,7,10,17,20 \mathrm{~nm}$, 然后生长碳 纳米管阵列. 当缓冲层厚度小于或等于 $4 \mathrm{~nm}$ 时, 得到较 高可纺性能的阵列. 三氧化二铝厚度的影响原因尚不清 楚. 此外, 在实验中发现当三氧化二铝层厚度降低时, 相同条件下得到的阵列高度也降低, 因此三氧化二铝缓 冲层厚度对于催化剂的活性也可能产生影响.

\subsection{2 生长参数的影响}

除了催化剂, 各项生长参数对阵列的可纺性也有重 要影响, 如生长前热处理过程影响着铁颗粒的形成, 而 反应气氛则影响着碳纳米管成核与生长过程以及无定 型碳的产生。

(1)气体通入顺序的影响

化学气相沉积法制备碳纳米管阵列中使用的气体 包括氩气、氢气、乙烯, 其中氩气作为载气提供惰性气 氛, 需一直保持通气. 氢气用于还原铁膜, 使铁膜裂解 成颗粒, 并使催化剂上沉积的无定形碳脱落 ${ }^{[57]}$. 乙烯作 为反应气体, 同时也具有还原性. 因此, 生长过程中氢 气与乙烯气体的通入顺序对于阵列的可纺性具有重大 影响 ${ }^{[58]}$. 如图 1 所示, 生长过程分为 4 个阶段.

我们发现，在升温过程中通入氢气与乙烯，制备的 阵列具有较好的可纺性. 若升温过程中只通入氢气而不 通乙烯, 到反应温度后再稳定一段时间, 铁膜会在升温 过程中先裂解成大小均匀的铁颗粒, 在达到反应温度后 的稳定阶段, 颗粒发生聚集, 数目变少而尺寸变大且不 均匀, 难以得到可纺的阵列. 通过扫描电镜观察升温到 不同温度点停止加热并冷却后催化剂纳米颗粒的形貌,

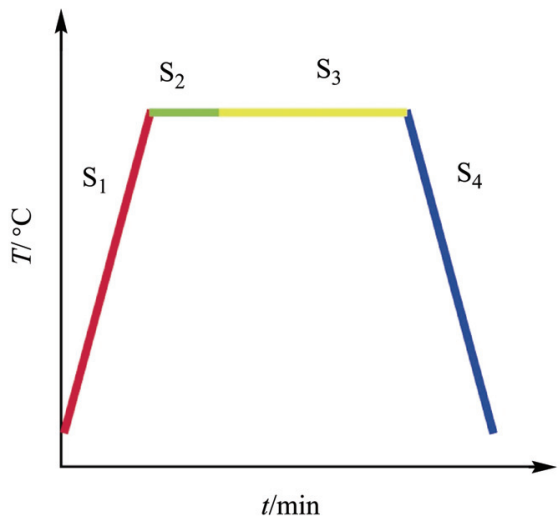

图 1 化学气相沉积法合成碳纳米管阵列 4 个主要阶段 $\left(\mathrm{S}_{1}\right.$ : 升温; $\mathrm{S}_{2}$ : 稳定; $\mathrm{S}_{3}$ : 生长; $\mathrm{S}_{4}$ : 降温)

Figure 1 Synthetic process of carbon nanotube (CNT) array by chemical vapor deposition $\left(\mathrm{S}_{1}\right.$ : ramping up; $\mathrm{S}_{2}$ : catalyst pretreatment; $\mathrm{S}_{3}$ : growth; $\mathrm{S}_{4}$ : cooling)

我们发现，铁膜在大约 $500{ }^{\circ} \mathrm{C}$ 时开始裂解成铁颗粒. 随 着温度的继续升高，铁颗粒数目不断增加(图 2). 为了制 备可纺的阵列, 在温度升至 $500{ }^{\circ} \mathrm{C}$ 前(铁膜开始裂解时) 就必须通入乙烯，使铁颗粒形成时乙烯分解，碳溶解在 铁颗粒中得到大小均匀、密度高且溶解有碳的铁颗粒, 进而获得高质量的碳纳米管阵列. 随着达到反应温度后 的稳定阶段时间 $\left(\mathrm{S}_{2}\right.$ 过程)变长, 铁颗粒分布变宽, 铁颗 粒的均匀性和密度也降低, 制备的碳纳米管往往不可 纺. 因此, 从升温开始就持续通入乙烯, 即不提供稳定 时间，得到的阵列具有最好的可纺性.

(2)升温速率、生长时间及反应温度的影响

如图 1 所示, 在常用的 $30 \sim 73{ }^{\circ} \mathrm{C} / \mathrm{min}$ 范围内改变 升温速率 $\left(\mathrm{S}_{1}\right.$ 过程), 没有发现阵列在可纺性方面有明显 区别. 因此在大多数情况下, 升温速率对催化剂颗粒裂 解的影响不大. 生长时间与反应温度对阵列可纺性具有 显著的影响. 可纺阵列要求碳纳米管表面尽量干净, 但 为了获得高性能的碳纳米管材料，又希望阵列尽可能 高, 因此要求更长的生长时间或/和更高的反应温度，这 样往往导致更多无定形碳等杂质的产生. 所以必须平衡 上述两个因素，找到最合适的生长时间与反应温度.

本论文所用催化剂活性较高, 可保持至少 $120 \mathrm{~min}$. 但生长时间超过 $15 \mathrm{~min}$ 后, 得到的阵列可纺性明显下 降, 这可能与阵列的表面形貌和结构等密切有关. 图 3 对比了不同生长时间的阵列侧面图. 当生长时间为 15 $\min$ 时阵列表面干净，具有良好的可纺性. 随着生长时 间的增加，阵列顶部出现无规的沉积层，无规层厚度随 着生长时间的增加而增加. 无规层可能导致碳纳米管在 纺丝过程中发生断裂，阵列可纺性降低(只能得到长度 小于 $1 \mathrm{~m}$ 的碳纳米管栅或纤维), 甚至不可纺(完全拉不 出连续的碳纳米管). 为了进一步验证上述结论, 我们 小心揭掉顶部无规层，发现剩余阵列未被破坏的部分仍 然具有可纺性，因此顶部无规碳层是阻碍阵列可纺性的 直接原因. 

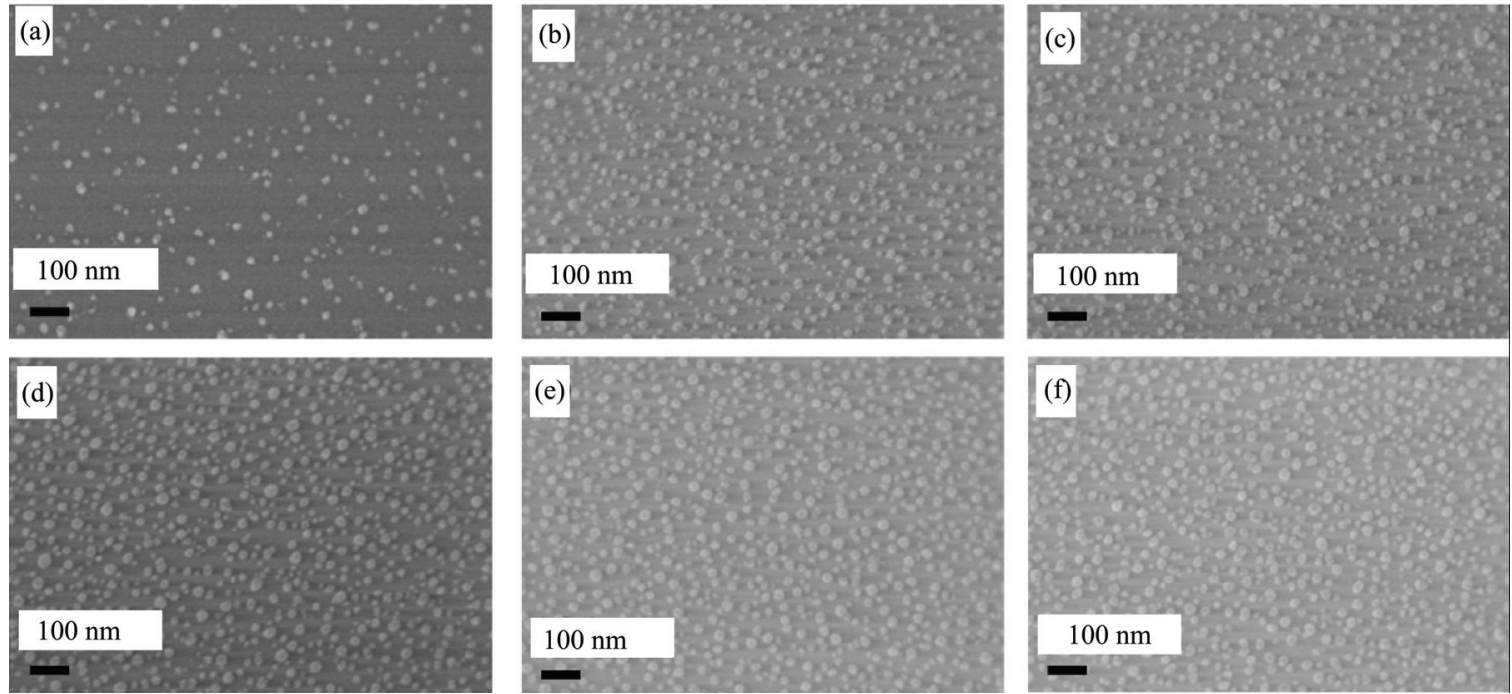

图 2 铁膜在不同温度下形成催化剂颗粒的扫描电镜照片

Figure 2 Scanning electron microscopy (SEM) images of the catalyst film treated under different temperatures (a) $500{ }^{\circ} \mathrm{C}$; (b) $550{ }^{\circ} \mathrm{C}$; (c) $600{ }^{\circ} \mathrm{C}$; (d) $650{ }^{\circ} \mathrm{C}$; (e) $700{ }^{\circ} \mathrm{C}$; (f) $750{ }^{\circ} \mathrm{C}$
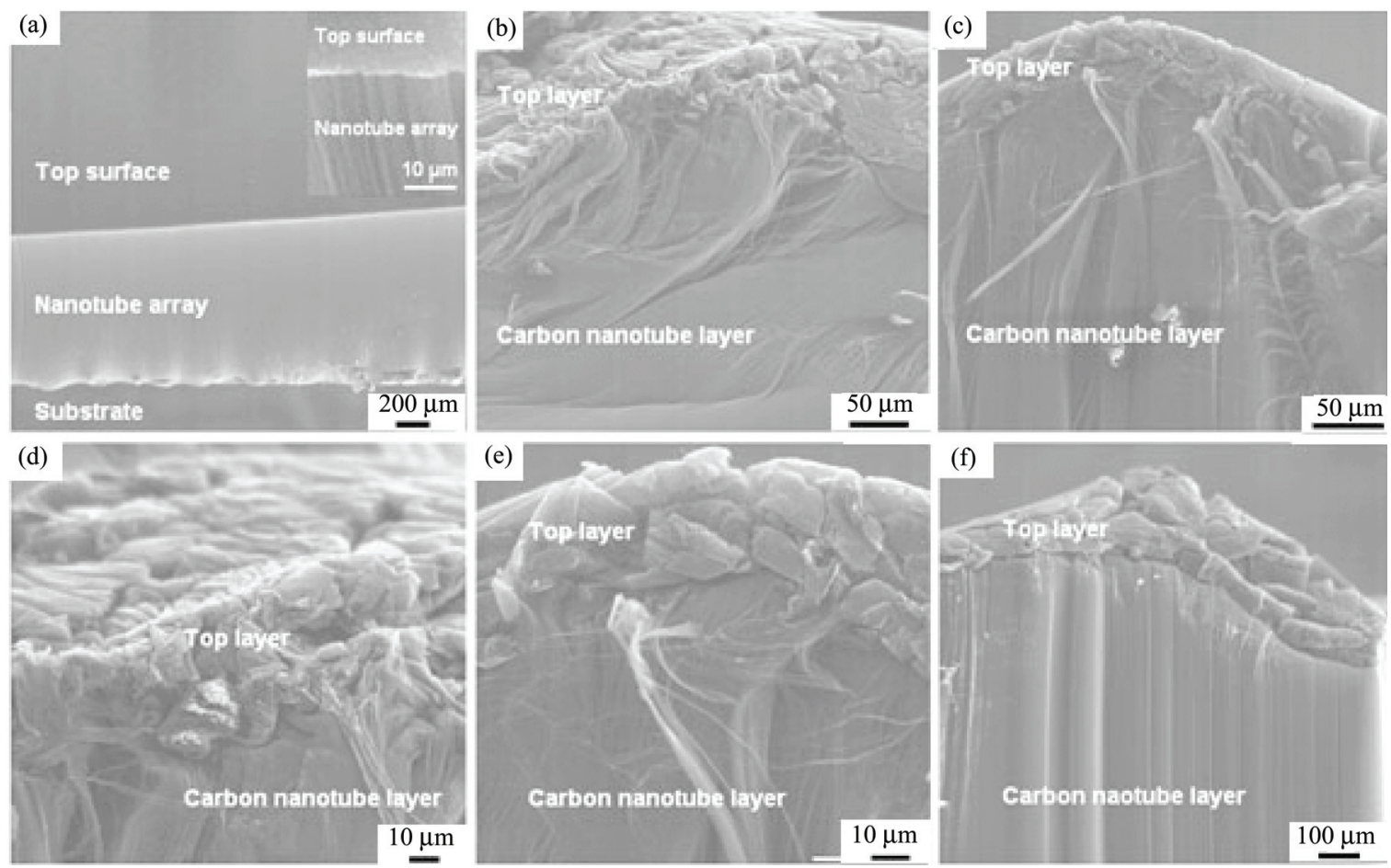

图 3 不同生长时间碳纳米管阵列侧面扫描电镜图

Figure 3 SEM images of CNT arrays with different growth times by a side view

(a) $15 \mathrm{~min}$ (inset, high magnification); (b) $40 \mathrm{~min}$; (c) $60 \mathrm{~min}$; (d) $90 \mathrm{~min}$; (e) $110 \mathrm{~min}$; (f) $120 \mathrm{~min}$

通过透射电镜对阵列顶部的无规层进行表征，图 4 显示了生长时间为 60,90 和 $120 \mathrm{~min}$ 时阵列顶部无规层 的透射电镜照片. 可以看出, 无规层中沉积物为无规、 薄片状结构的聚集体, 其尺寸随着生长时间的增加而下 降. 如当生长时间为 $60 \mathrm{~min}$ 时, 聚集体大于 $200 \mathrm{~nm}$; 当 生长时间为 $120 \mathrm{~min}$ 时聚集体小于 $200 \mathrm{~nm}$. 高分辨透射 电镜(图 4d)进一步发现聚集体主要为无定形碳和石墨, 同时含有少量碳纳米管.
生长温度对碳纳米管阵列可纺性的影响与生长时 间相似. 生长温度越高时生长速率越快, 同时阵列顶部 无定形碳的沉积速率也越快. 生长温度越低时生长速率 越慢, 无定形碳沉积速率也越低, 如果要获得高的阵列 则必须更长的时间. 因此需要在阵列的高度与可纺性之 间找到合适的平衡点. 本论文研究了生长温度为 730 , $740,745,750,755,760$ 和 $770{ }^{\circ} \mathrm{C}$ 的情况，发现反应温度 $740{ }^{\circ} \mathrm{C}$ 最适合. 

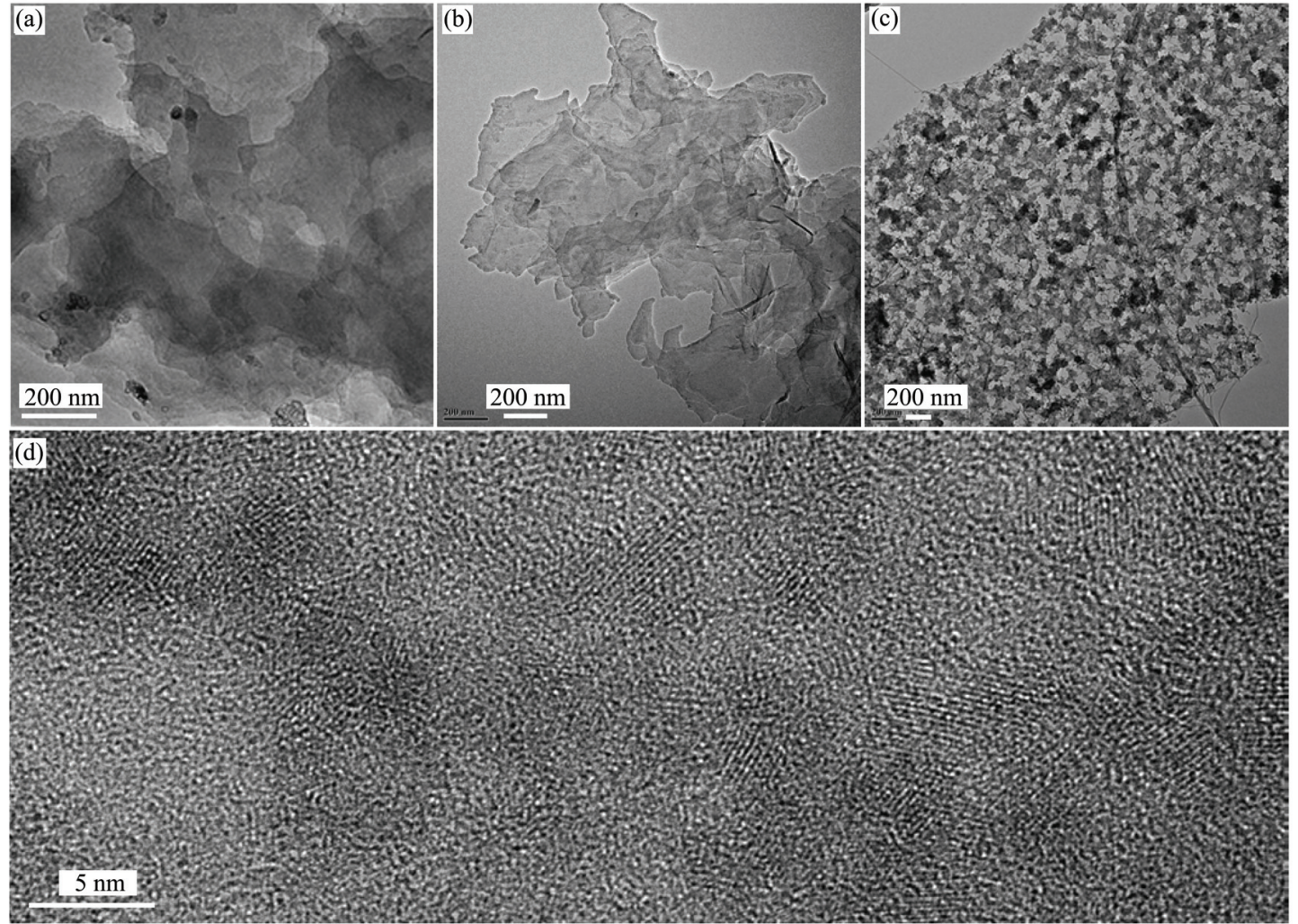

图 4 碳纳米管阵列顶部无规层的透射电镜图

Figure 4 Transmission electron microscopy (TEM) images on the top surface of CNT arrays with different growth times (a) $60 \mathrm{~min}$; (b) $90 \mathrm{~min}$; (c) $120 \mathrm{~min}$; (d) high resolution TEM image at the growth time of $120 \mathrm{~min}$

(3)反应气体比例和总流量的影响

在内管径 $45 \mathrm{~mm}$, 加热区长 $40 \mathrm{~cm}$ 的管式炉中, 改 变乙烯比例为 $5 \% \sim 50 \%$, 氢气比例 $3 \% \sim 17 \%$, 总流量 $200 \sim 1040 \mathrm{~cm}^{3} / \mathrm{min}$ 之间, 发现最佳的气体流量比例为 氩气：乙烯：氢气 $=40: 9: 3$, 总流量为 $400 \sim 520$ $\mathrm{cm}^{3} / \mathrm{min}$ (标准状态下气体体积流量, 下同)比较合适. 对 于更小的内径 $24.5 \mathrm{~mm}$ 的管式炉, 总流量 $260 \mathrm{~cm}^{3} / \mathrm{min}$ 比较合适. 在较宽的流量范围内都可以制备可纺的阵 列，阵列的可纺性对于总流量变化不敏感.

综上所述, 为了得到可纺的碳纳米管阵列, 制备催 化剂的最佳参数为: 腔体初始压力 $5 \times 10^{-4} \mathrm{~Pa}$, 三氧化 二铝沉积速率 $2.0 \AA / \mathrm{s}$ 、最终厚度 $3 \mathrm{~nm}$, 铁沉积速率 0.5 $\AA / \mathrm{s}$ 、最终厚度 $1.2 \mathrm{~nm}$. 对于直径 $45 \mathrm{~mm}$ 的管式炉, 生长 可纺阵列的最佳条件为: 升温开始时即通入乙烯和氢 气, 氩气、乙烯、氢气的流量分别为 $400 、 90 、 30 \mathrm{~cm}^{3} / \mathrm{min}$, 生长温度 $740{ }^{\circ} \mathrm{C}$, 生长时间 $10 \mathrm{~min}$.

\subsection{3 碳纳米管阵列的表征}

碳纳米管阵列主要通过扫描和透射电子显微镜来 表征, 图 5 是典型照片, 阵列高度在 $200 \mu \mathrm{m}$ 左右. 从阵 列的侧面图(图 $5 \mathrm{~b}$ 和 $5 \mathrm{c}$ )中可以看出, 碳纳米管取向排 列, 顶部与底部都很干净, 没有无定型碳的沉积. 从透 射电镜(图 5d)中可以看出, 碳纳米管为多壁结构, 管径 约 $10 \mathrm{~nm}$. 虽然碳纳米管的壁数和直径是影响碳纳米管
栅和纤维的重要参数, 目前仍然很难精确控制可纺碳纳 米管的壁数和直径. 碳纳米管纤维重量密度为 0.543 $\mathrm{g} / \mathrm{cm}^{3}$; 碳纳米管柱的面密度为 $1.41 \mu \mathrm{g} / \mathrm{cm}^{2}$. 从可纺的 碳纳米管阵列中得到碳纳米管栅和纤维的细节请参考 实验部分.

\section{2 取向碳纳米管/高分子复合阵列}

通过把高分子溶液或者高分子熔体加入碳纳米管 阵列中, 可以方便、高效率制备复合阵列, 下面以取向 碳纳米管/环氧树脂为例来说明，通过溶液过程制备. 首 先按照环氧树脂配方 “EPON 812” 制备包埋原液 ${ }^{[29]}$, 包 埋液主要包括环氧树脂(SPI-Pon 812)、十二烯基丁二酸 酐、甲基纳迪克酸䣶、固化促进剂 2,4,6-三(二甲氨基甲 基)苯酚等. 然后把碳纳米管阵列浸泡在包埋液中, 环 氧树脂通过渗透进入到阵列的碳纳米管间隙中. 最后, 在聚合箱中常压下 $60{ }^{\circ} \mathrm{C}$ 固化 $36 \mathrm{~h}$, 即可得到用环氧树 脂包埋好的碳纳米管阵列. 图 6 为典型的复合阵列侧面 扫描电镜照片, 碳纳米管保持高度取向排列. 我们重点 研究复合阵列的电学性能, 结果发现, 沿碳纳米管取向 方向的导电率在 $10^{2} \sim 10^{3} \mathrm{~S} / \mathrm{cm}$, 而垂直于碳纳米管取 向方向的导电率最高可达 $10^{2} \mathrm{~S} / \mathrm{cm}$. 作为对比, 通过传 统溶液共混制备的无规取向碳纳米管/环氧树脂复合阵 列的导电率在 $10^{-6} \sim 10^{-3} \mathrm{~S} / \mathrm{cm}$. 

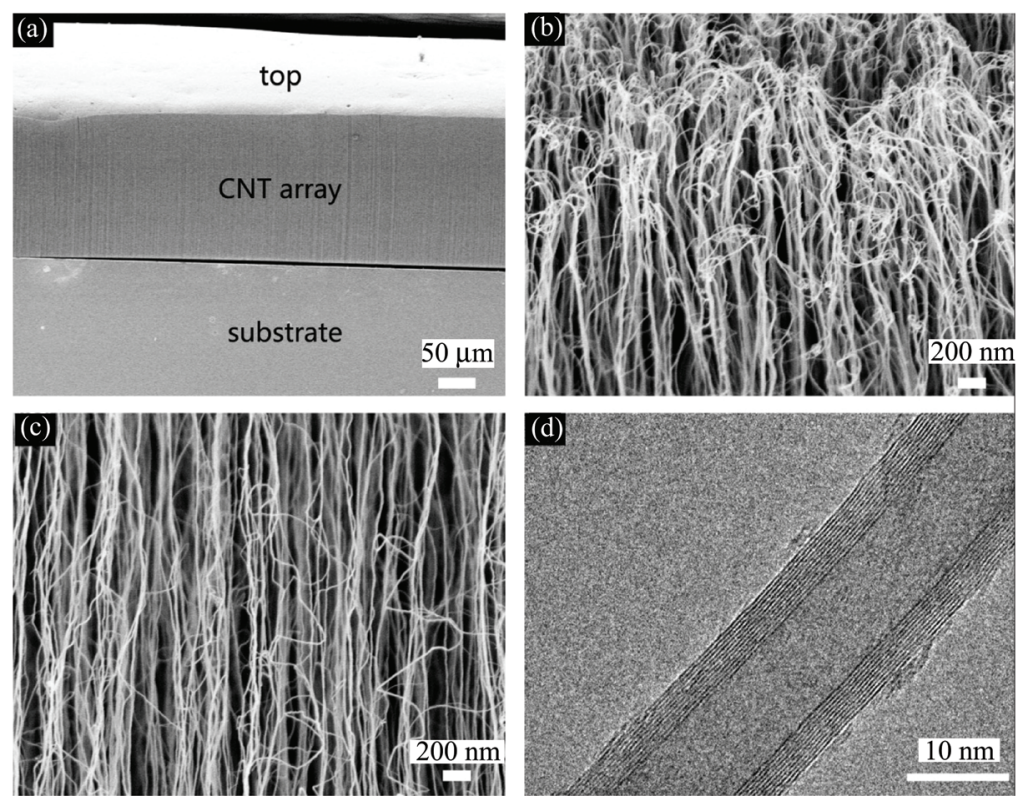

图 5 可纺碳纳米管阵列扫描和透射电镜图: (a)侧面扫描电镜图; (b)顶部高分辨扫描电镜图; (c)底部高分辨扫描电镜图; (d)碳纳米管高分辨透射电 镜图

Figure 5 SEM and TEM images of spinnable CNT array: (a) SEM image by a side view; (b) high resolution SEM image of the top part; (c) high resolution SEM image of the bottom part; (d) high resolution TEM of a CNT

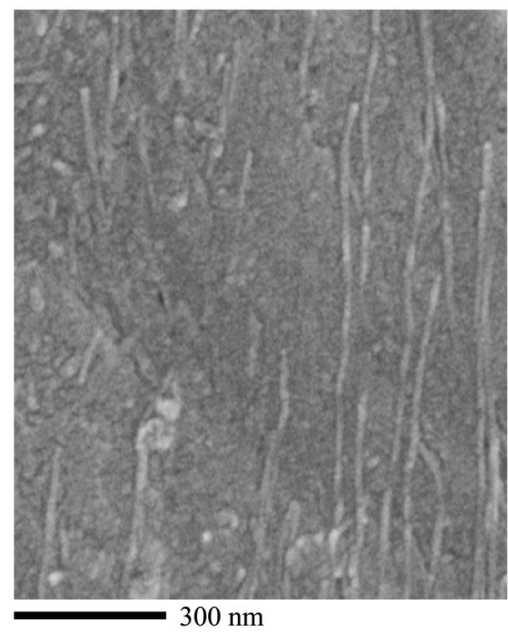

图 6 取向碳纳米管/环氧树脂复合阵列侧面扫描电镜图

Figure 6 SEM image of an aligned CNT/epoxy composite array by a side view

\section{3 取向碳纳米管/高分子复合膜}

高分子可以通过溶液或熔融过程加入到碳纳米管 栅中制备取向碳纳米管/高分子复合材料, 下面以侧链 含有偶氮苯液晶基元的高分子为例. 图 7a 是通过熔融 法制备液晶高分子复合膜的示意图, 碳纳米管栅首先固 定在玻璃片上, 制成液晶盒, 然后把液晶单体、交联剂 和引发剂(结构式参考支持信息图 S1 和 S2)的熔融混合 物按比例加入到液晶盒中, 原位光聚合制备复合薄膜. 由于碳纳米管优异的机械性能, 复合材料(碳纳米管重 量含量为 $0.26 \%$ )的机械强度比纯液晶材料提高了一倍 (图 8). 另外, 纯的液晶材料是不导电的, 而碳纳米管赋

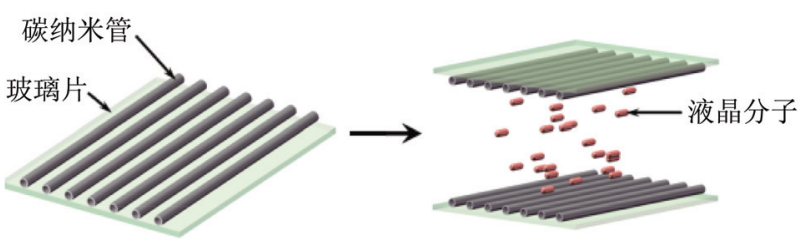

图 7 取向碳纳米管/高分子复合膜制备示意图

Figure 7 Schematic illustration to the synthesis of the aligned CNT/polymer composite film

予复合材料优异的导电性能，其中沿碳纳米管取向方向 的导电率最高可以达到 $10^{2} \mathrm{~S} / \mathrm{cm}$, 良好的导电性能可以 避免其他液晶材料中存在的静电聚集问题.

需要指出的是, 对于含有偶氮苯基元的侧链型液晶 弹性体，偶氮苯基元在紫外光照射下从反式转变为顺式 结构, 停止紫外光照后, 在可见光照射下, 顺式又回复 到反式结构. 一般来说，偶氮苯材料对 $360 \mathrm{~nm}$ 左右的光 吸收非常强, 紫外光照射下, $99 \%$ 以上的光被表层 $1 \mu \mathrm{m}$ 以内的偶氮苯吸收, 发生反式一顺式异构化, 其他部分 的偶氮苯依然保持反式结构 ${ }^{[33]}$. 而偶氮苯复合薄膜的 厚度为 $30 \mu \mathrm{m}$, 发生异构化的偶氮苯量约为 $3.3 \%$, 由于 材料表面的收缩导致薄膜材料的向光弯曲. 可见光照射 下，发生异构化的偶氮苯分子恢复到反式结构, 复合薄 膜也恢复到平展状态. 从而可以实现薄膜材料的可逆光 致形变, 这种可逆变形只发生在液晶薄膜中液晶基元的 取向方向. 液晶取向的传统方法通过机械摩擦来实现, 容易引入材料碎片、产生静电、对材料结构产生破坏 ${ }^{[59,60]}$. 如果以高度取向碳纳米管桶作为模板, 实现液晶基元沿 碳纳米管长度方向取向, 可以有效解决上述问题. 由于 


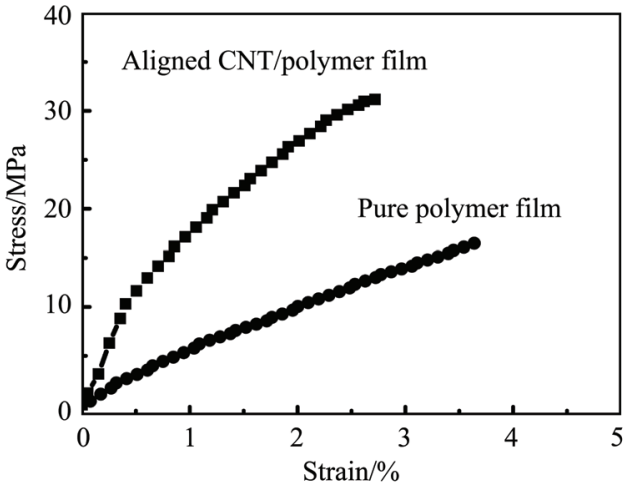

图 8 纯液晶高分子膜和取向碳纳米管/夜晶高分子复合膜的应力-应 变曲线

Figure 8 Stress-strain curves of pure liquid polymer and aligned CNT/liquid polymer composite films

液晶基元沿碳纳米管长度方向取向, 在紫外光照射下复 合膜沿碳纳米管长度方向向光弯曲, 接着在可见光照射 下又回复到原来状态. 另外, 引入取向碳纳米管不但大 幅度提高液晶高分子材料的机械强度和赋予其导电性, 同时也大大提高液晶材料的稳定性, 上述可逆形变重复 上百次没有明显衰减.

\section{4 取向碳纳米管/高分子复合纤维}

在碳纳米管纤维中, 碳纳米管之间通过较弱的范德 华力连接起来, 在较低拉力下即发生滑移, 导致纤维机 械强度较低, 远低于单根碳纳米管强度. 从实验结果与 文献报道来看, 干法纺丝制备的取向碳纳米管纤维强度 在 300 500 MPa 左右. 通过纺丝过程或纺丝后对纤维 进行合适的处理, 纤维强度进一步增加到 $1 \sim 1.5$ $\mathrm{GPa}^{[40,49,54,61 \sim 71]}$. 主要的处理方法包括: 纺丝过程中纤 维通过挥发性溶剂, 如乙醇、水、丙酮等 ${ }^{[49,67,68]}$; 纺丝过 程中对纤维施加拉伸应力, 提高纤维内碳纳米管的取向 度 ${ }^{[63]}$; 对所得到的纤维进行热处理 ${ }^{[63,71]}$; 对所得到的纤 维进行再次加捻, 增加碳纳米管之间的摩擦力 ${ }^{[61,65,68]}$. 总结来说, 影响碳纳米管纤维强度的因素包括碳纳米管 长度 ${ }^{[61]}$ 、管径和壁数 ${ }^{[68]}$, 碳纳米管在纤维内部的堆积取 向 ${ }^{[63,68]}$, 碳纳米管纤维的直径 ${ }^{[68,74,75]}$, 碳纳米管的缺陷 与杂质的含量.

如果引入高分子制备复合纤维, 高分子链可以缠结 在碳纳米管之间, 起到类似交联的效果, 有效防止碳纳 米管之间滑移, 复合纤维的机械强度将大大提高, 超过 纯碳纳米管纤维 ${ }^{[35,72,73]}$. 另外, 因为纤维的电阻主要来 自于碳纳米管之间的接触电阻, 而高分子链的缠结和交 联作用使相邻碳纳米管更加紧密接触, 降低接触电阻, 所以复合纤维的电导率也可以进一步提高. 以碳纳米管/ 聚丙烯酸复合纤维为例, 我们首先把纯碳纳米管纤维浸 泡在丙烯酸单体和交联剂的混合溶液中, 丙烯酸单体比 聚丙烯酸更能有效和均匀地形成复合纤维, 然后通过原 位热聚合得到碳纳米管/交联聚丙烯酸复合纤维. 图 9a
和 $9 \mathrm{~b}$ 分别为纯碳纳米管纤维和碳纳米管/交联聚丙烯酸 复合纤维的扫描电镜照片. 很明显, 复合后纤维直径由 $8.6 \mu \mathrm{m}$ 降低为 $7.2 \mu \mathrm{m}$. 虽然在较低放大倍数下复合纤维 与纯碳纳米管纤维看起来非常相似, 但二者的微观结构 完全不同. 图 9c 和 $9 \mathrm{~d}$ 比较了二者的高分辨扫描电镜照 片, 可以看出与纯碳纳米管纤维相比, 复合纤维中碳纳 米管束的表面更为光滑, 碳纳米管管束的平均直径由约 $28 \mathrm{~nm}$ 增加到 $52 \mathrm{~nm}$, 同时碳纳米管束的间距也有所下 降, 这是由于高分子吸附或缠结在碳纳米管表面所致. 图 9e 和 9f 为进一步比较单根碳纳米管的高分辨透射电 镜照片, 可以看出纯纤维中碳纳米管的直径约为 $12 \mathrm{~nm}$, 而复合纤维中碳纳米管表面吸附有约 $2.5 \mathrm{~nm}$ 厚的高分 子层. 通过对比碳纳米管纤维复合前后质量变化, 可以 计算出复合纤维中聚丙烯酸和碳纳米管的质量比为 $0.18 ： 1$. 接着我们研究了碳纳米管纤维复合前后机械 和电学性能的变化. 结果发现, 与传统方法制备的无规 碳纳米管/高分子复合纤维相比, 机械强度和导电率分 别提高了一个和三个数量级 ${ }^{[13]}$. 即与纯碳纳米管纤维 相比, 复合纤维的拉伸强度提高了约三倍, 达到 800 $\mathrm{MPa}$ (图 10); 导电率提高了约 1.2 倍, 达到 $400 \mathrm{~S} / \mathrm{cm}$. 作 为对比, 我们还研究了水处理后的碳纳米管纤维和碳纳 米管/未交联聚丙烯酸复合纤维的强度(图 10). 水处理 后的碳纳米管纤维直径减小 $10 \%$ 左右, 强度为 $300 \mathrm{MPa}$, 这是因为溶剂挥发导致碳纳米管之间接触更加紧密, 从 而提高纤维强度. 碳纳米管/未交联聚丙烯酸复合纤维 的强度为 $580 \mathrm{MPa}$, 低于交联后的 $800 \mathrm{MPa}$, 因为聚合 物交联后可以更加有效地防止相邻碳纳米管之间滑移.

\section{5 取向碳纳米管/高分子复合材料在太阳能电池中的 应用}

对于制备的取向碳纳米管/环氧树脂复合阵列(2.2 节所示), 进一步沿垂直于碳纳米管长度方向切片, 得 到取向碳纳米管/聚合物复合膜, 碳纳米管垂直于复合 膜. 图 11a 为复合膜表面扫描电镜图, 可以看到碳纳米 管被切断, 管口打开富集在膜的表面, 并且比较均匀地 分布. 因为在复合阵列中碳纳米管的长度在 $2 \sim 3 \mathrm{~mm}$, 而切片后复合膜主要控制在 $50 \mathrm{~nm}$ 到 $100 \mu \mathrm{m}$ 之间, 考 虑到碳纳米管在阵列中高度取向排列, 因此复合膜中绝 大部分碳纳米管上下贯通. 单根碳纳米管的导电率在 $10^{5} \mathrm{~S} / \mathrm{cm}$, 所以复合膜在垂直方向具有极高的导电率, 通过现有的表征技术尚难精确测定，理论计算发现其导 电率在 $10^{4} \mathrm{~S} / \mathrm{cm}$ 以上. 另外, 因为复合膜表面富集大量 开口的碳纳米管，而对于碳纳米管，开口的末端比管体 具有高得多的催化活性. 取向碳纳米管/高分子复合材 料还具有良好的柔性和其他优异的机械性能, 如较高的 强度, 因此可广泛用作一大类新型的柔性电极材料.

本论文重点以垂直取向碳纳米管/环氧树脂复合膜 取代铂作为对电极, 构建柔性染料敏化太阳能电池, 结 果发现得到的太阳能电池显示比较优异的性能. 图 $11 \mathrm{~b}$ 

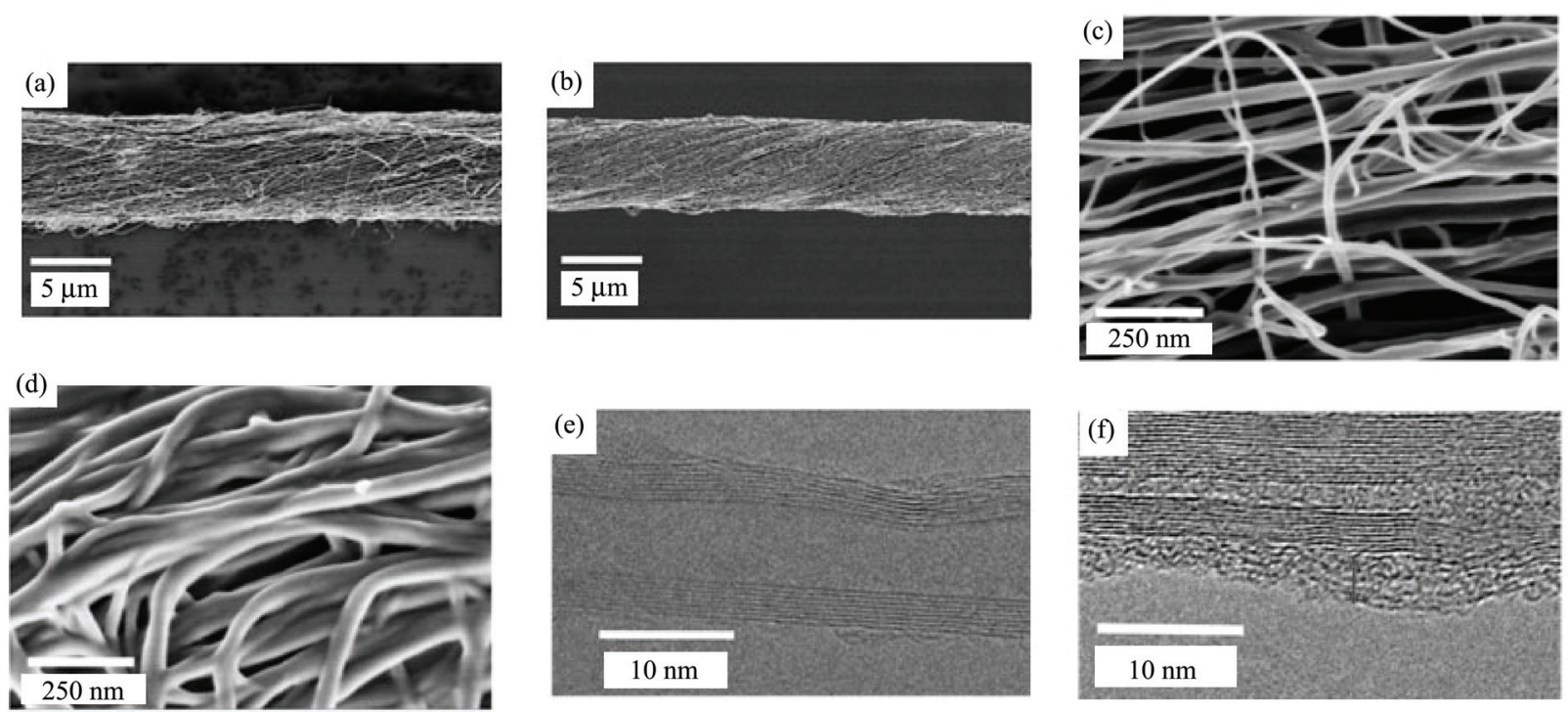

图 9 (a)纯碳纳米管纤维的扫描电镜图; (b)取向碳纳米管/聚丙烯酸复合纤维的扫描电镜图; (c)纯碳纳米管纤维的高分辨扫描电镜; (d)取向碳纳米 管/聚丙烯酸复合纤维的高分辨扫描电镜图; (e)纯碳纳米管的高分辨透射电镜图; (f)复合聚丙烯酸的碳纳米管的高分辨透射电镜照片, 其中红色的 箭头显示高分子的厚度约为 $2.5 \mathrm{~nm}$

Figure 9 (a) SEM image of pure CNT fiber; (b) SEM image of aligned CNT/poly(acrylic acid) composite fiber; (c) high resolution SEM image of a pure CNT fiber; (d) high resolution SEM image of aligned CNT/poly(acrylic acid) composite fiber; (e) high resolution TEM image of a pure CNT; (f) high resolution TEM image of a CNT attached with poly(acrylic acid). The arrow indicates the thickness of polymer layer to be about $2.5 \mathrm{~nm}$

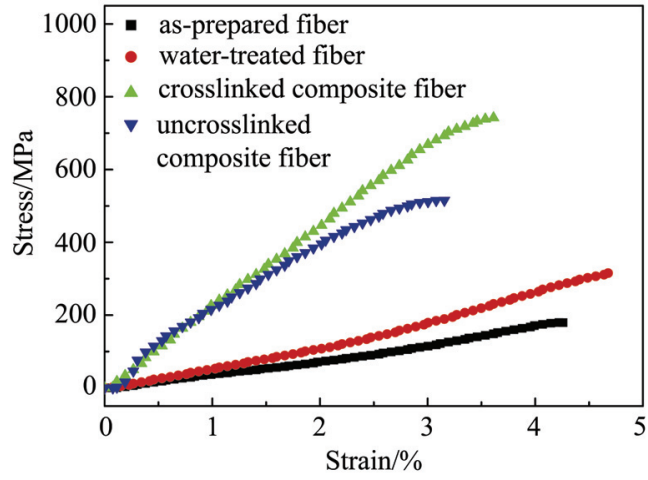

图 10 直接制备的碳纳米管纤维(a)、水处理后碳纳米管纤维(b)、取 向碳纳米管/未交联聚丙烯酸复合纤维(c)以及取向碳纳米管/交联聚丙 烯酸复合纤维(d)的应力-应变曲线

Figure 10 Stress-strain curves of the as-spun CNT fiber (a), the pure CNT fiber after being treated by water (b), and the aligned CNT/poly(acrylic acid) composite fiber without (c) and with (d) being cross-linked

为 $10 \mu \mathrm{m}$ 厚复合膜作为对电极的典型电流-电压曲线, 开路电压、短路电流密度、填充因子和光电转换效率分 别为 $0.70 \mathrm{~V} 、 11.18 \mathrm{~mA} / \mathrm{cm}^{2} 、 0.25$ 和 $1.96 \%$. 虽然光电 转化效率还不够理想, 但通过优化复合膜电极的表面结 构和提高电池的构建工艺, 光电转化效率可以进一步提 高.

同时, 对于平行取向的碳纳米管/高分子复合膜, 同 样可以作为对电极构建柔性染料敏化太阳能电池. 图 $12 \mathrm{a}$ 为用于构建电池复合膜典型扫描电镜照片, 因为碳 纳米管在平行方向取向排列, 沿碳纳米管取向方向电导 率较高. 但在复合膜的垂直方向, 因为碳纳米管之间接
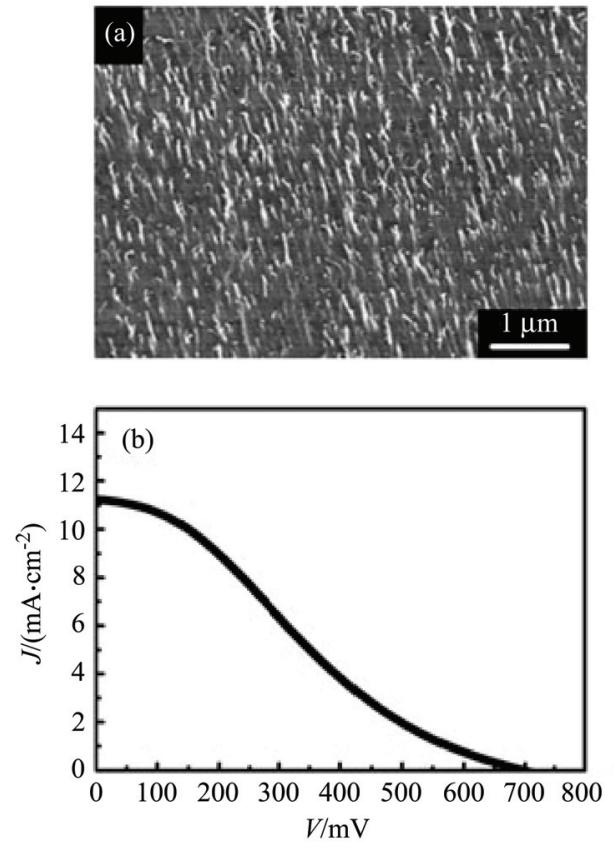

图 11 (a)垂直取向碳纳米管/聚合物复合薄膜表面的扫描电镜图; (b) 以垂直取向碳纳米管/聚合物复合薄膜作为对电极所构建的染料敏化 太阳能电池在 AM1.5 下测得的 $J-V$ 曲线

Figure 11 (a) SEM image of the surface of a vertically aligned $\mathrm{CNT} /$ polymer composite film; (b) $J-V$ curve of a dye-sensitized solar cell by using the vertically aligned CNT/polymer composite film as a counter electrode measured under AM1.5

触电阻较大，因此作为电极材料其效率不如垂直取向的 碳纳米管/高分子复合膜. 对于 $10 \mu \mathrm{m}$ 厚的复合膜，在同 等条件下, 开路电压、短路电流密度、填充因子和光电 
转换效率分别是 $0.67 \mathrm{~V} 、 3.45 \mathrm{~mA} / \mathrm{cm}^{2} 、 0.26$ 和 $0.61 \%$ (图 $12 b)$.
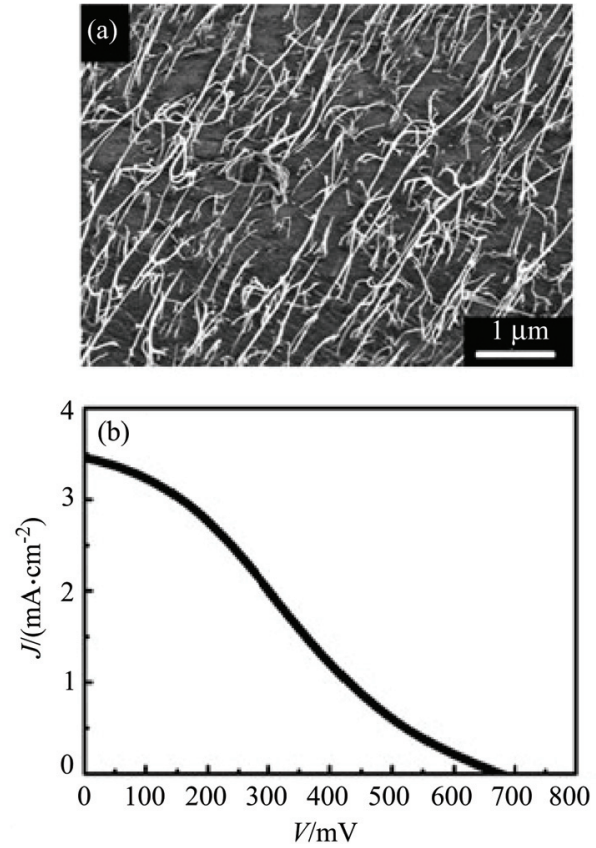

图 12 (a)平行取向碳纳米管/聚合物复合薄膜表面的扫描电镜图; (b) 以平行取向碳纳米管/聚合物复合薄膜作为对电极所构建的染料敏化 太阳能电池在 AM1.5 下测得的 $J-V$ 曲线

Figure 12 (a) Surface of horizontal aligned CNT/polymer composite film; (b) $J-V$ curve of DSSC using the horizontal aligned CNT/polymer composite film as counter electrode under AM1.5

\section{3 结论}

本论文制备了一系列取向碳纳米管/高分子复合阵 列、复合膜和复合纤维, 制备的关键是通过化学气相沉 积法合成具有可纺性的高质量碳纳米管阵列, 因此比较 系统地探讨催化剂以及合成参数等实验条件对碳纳米 管阵列可纺性的影响. 对于电子束蒸发沉积催化剂, 我 们发现腔体压力和蒸镀速率对催化剂质量具有重要影 响, 从而决定碳纳米管阵列的可纺性. 通过系统研究, 总结出制备催化剂的最佳参数为: 腔体初始压力 $5 \times$ $10^{-4} \mathrm{~Pa}$, 三氧化二铝沉积速率 $2.0 \AA \mathrm{A} / \mathrm{s}$ 、最终厚度 $3 \mathrm{~nm}$, 铁沉积速率 $0.5 \AA / \mathrm{s}$ 、最终厚度 $1.2 \mathrm{~nm}$. 另外, 我们还发 现催化剂厚度、气体通入顺序、升温速率、生长时间、 反应温度、反应气体比例和流量等合成参数对得到的阵 列可纺性也具有明显影响. 对于直径 $45 \mathrm{~mm}$ 的管式炉, 生长可纺阵列的最佳条件为: 升温开始时即通入乙烯和 氢气, 氩气、乙烯、氢气的流量分别为 $400 、 90 、 30$ $\mathrm{cm}^{3} / \mathrm{min}$, 生长温度 $740{ }^{\circ} \mathrm{C}$, 生长时间 $10 \mathrm{~min}$. 在获得可 纺碳纳米管阵列的基础上, 通过干法纺丝得到取向的碳 纳米管栅和纤维, 最后通过溶液或熔融方法引入高分子 制备复合材料, 本论文中高分子主要是物理吸附在碳纳 米管表面. 因为碳纳米管高度取向排列, 复合材料显示 优异的物理性能, 如与无规碳纳米管/高分子复合材料 相比, 机械强度和导电率可分别提高一个和三个数量
级，因此新型复合材料在航空、航天、汽车、能源等广 泛领域显示重要的应用前景. 本论文主要探讨其作为电 极在有机太阳能电池中的应用.

\section{4 实验部分}

本实验使用催化剂基底为表面含有 $1 \mu \mathrm{m}$ 厚二氧化 硅(p 型, 100)的硅片, 三氧化二铝和铁膜通过电子束蒸 发先后沉积在二氧化硅层上. 为了对比不同真空蒸镀对 于催化剂膜质量的影响, 本实验还使用电阻热蒸镀沉积 铁膜. 实验发现, 当使用相同沉积速率时, 得到的催化 剂对阵列的可纺性影响不大, 因此本论文主要使用电子 束蒸发方式制备催化剂薄膜.

化学气相沉积法典型的反应过程为: 催化剂平放入 管式炉中，首先往管式炉中通氩气以排除其中的空气， 然后通入载气氩气、氢气和乙烯气体, 接着升温至设定 的反应温度, 反应 $10 \mathrm{~min}$ 后, 停止通入氢气与乙烯, 在 氩气氛围中降至室温.

液晶单体、交联剂和引发剂由俞燕蕾教授课题组提 供. 纤维的纺丝收集过程如图 S3 和 S4(见支持信息)所 示. 首先把可纺碳纳米管阵列固定在样品台上, 样品台 可以旋转以实现纤维加捻; 然后使用刀片从阵列边缘拉 出连续的碳纳米管栅, 并将碳纳米管栅加捻形成纤维后 固定在用于收集的辊筒上. 然后打开控制样品台的电 机, 使样品以 $2000 \mathrm{r} / \mathrm{min}$ 转动, 同时打开控制辊筒的电 机, 从而连续拉出碳纳米管纤维, 拉伸速率为 15 $\mathrm{cm} / \mathrm{min}$. 若不加捻, 则得到连续的取向碳纳米管栅.

\section{References}

[1] Zhao, Y. L.; Stoddart, J. F. Acc. Chem. Res. 2009, 42, 1161.

[2] Harris, P. J. F. Int. Mater. Rev. 2004, 49, 31.

[3] Peng, H.; Chen, D.; Huang, J.; Chikkannanavar, S. B.; Hanisch, J.; Peterson, D. E.; Doorn, S. K.; Lu, Y.; Zhu, Y.; Jia, Q. Phys. Rev. Lett. 2008, 101, 145501.

[4] Peng, H.; Zhu, Y.; Peterson, D. E.; Lu, Y. Adv. Mater. 2008, 20, 1199.

[5] Shi, D. Adv. Funct. Mater. 2009, 19, 3356

[6] Liu, Z.; Tabakman, S. M.; Chen, Z.; Dai, H. J. Nat. Protoc. 2009, 4, 1372.

[7] Guldi, D. M.; Rahman, G. M. A.; Zerbetto, F.; Prato, M. Acc. Chem. Res. 2005, 38, 871.

[8] Kondratyuk, P.; Yates, J. T. Acc. Chem. Res. 2007, 40, 995.

[9] Huang, J. Y.; Ding, F.; Jiao, K.; Yakobson, B. I. Small 2007, 3, 1735.

[10] Feldman, A. K.; Steigerwald, M. L.; Guo, X. F.; Nuckolls, C. Acc. Chem. Res. 2008, 41, 1731.

[11] Iijima, S. Nature 1991, 354, 56.

[12] Ajayan, P. M.; Tour, J. M. Nature 2007, 447, 1066.

[13] Wang, B.; Sun, G. B.; Sun, G. E.; He, X. F.; Liu, J. J. Acta Polym. Sinica 2003, (3), 408. (王彪, 孙广平, 孙国恩, 何晓峰, 刘景江, 高分子学报, 2003, (3), 408.)

[14] Feng, H. Z.; Wang, X. L.; Xia, H. S. Acta Polym. Sinica 2009, (9), 953. (冯桓榰, 王雪力, 夏和生, 高分子学报, 2009, (9), 953.)

[15] Bryning, M. B.; Milkie, D. E.; Islam, M. F.; Kikkawa, J. M.; Yodh, A. G. Appl. Phys. Lett. 2005, 87, 161909.

[16] Badaire, S.; Poulin, P.; Maugey, M.; Zakri, C. Langmuir 2004, 20, 10367.

[17] Liu, T.; Phang, I. Y.; Shen, L.; Chow, S. Y.; Zhang, W.-D. Macromolecules 2004, 37, 7214.

[18] Bhattacharyya, A. R.; Sreekumar, T. V.; Liu, T.; Kumar, S.; Ericson, L. M.; Harge, R. H.; Smalley, R. E. Polymer 2003, 44, 2373. 
[19] Du, F.; Guthy, C.; Kashiwagi, T.; Fischer, J. E.; Winey, K. I. J. Polym. Sci., Part B: Polym. Phys. 2006, 44, 1513.

[20] Zhu, J.; Peng, H.; Rodriguez-macias, F.; Margrave, J. L.; Khabashesku, V. N.; Iman, A. M.; Lozano, K.; Barrera, E. V. Adv. Funct. Mater. 2004, 14, 643 .

[21] Kimura, T.; Ago, H.; Tobita, M.; Ohshima, S.; Kyotani, M.; Yumura, M. Adv. Mater. 2002, 14, 1380 .

[22] Jin, L.; Bower, C.; Zhou, O. Appl. Phys. Lett. 1998, 73, 1197.

[23] Safadi, B.; Andrews, R.; Grulke, E. A. J. Appl. Polym. Sci. 2002, 84, 2660.

[24] Haggenmueller, R.; Zhou, W.; Fischer, J. E.; Winey, K. I. Nanosci. Nanotechnol. 2003, 3, 105

[25] Ge, J. J.; Hou, H.; Li, Q.; Graham, M. J.; Greiner, A.; Reneker, D. H.; Harris, F. W.; Cheng, S. Z. D. J. Am. Chem. Soc. 2004, 126 , 15754.

[26] Gao, J.; Yu, A.; Itkis, M. E.; Bekyarova, E.; Zhao, B.; Niyogi, S.; Haddon, R. C. J. Am. Chem. Soc. 2004, 126, 16698.

[27] Ko, F.; Gogotsi, Y.; Ali, A.; Naguib, N.; Ye, H.; Yang, G.; Li, C.; Willis, P. Adv. Mater. 2003, 15, 1161.

[28] Peng, H. S.; Sun, X. M.; Cai, F. J.; Chen, X. L.; Zhu, Y. C.; Liao, G.; Chen, D. Y.; Li, Q. W.; Lu, Y. F.; Zhu, Y. T.; Jia, Q. X. Nat. Nano 2009, 4, 738 .

[29] Huang, S. Q.; Li, L.; Yang, Z. B.; Zhang, L. L.; Saiyin, H.; Chen, T. Adv. Mater. 2011, 23, 4707.

[30] Li, L.; Yang, Z. B.; Gao, H. J.; Zhang, H.; Ren, J.; Sun, X. M.; Chen, T.; Kia, H. G.; Peng, H. S. Adv. Mater. 2011, 23, 3730.

[31] Guo, W. H.; Liu, C.; Sun, X. M.; Yang, Z. B.; Kia, H. G.; Peng, H. S. J. Mater. Chem. 2012, 12, 903.

[32] Peng, H. S. J. Am. Chem. Soc. 2008, 130, 42.

[33] Wang, W.; Sun, X. M.; Wu, W.; Peng, H. S.; Yu, Y. L. Angew. Chem. Int. Ed. 2012, 51, 4644 .

[34] Liu, K.; Sun, Y. H.; Lin, X. Y.; Zhou, R. F.; Wang, J. P.; Fan, S. S.; Jiang, K. L. ACS Nano 2010, 4, 5827.

[35] Fang, C.; Zhao, J. N.; Jia, J. J.; Zhang, Z. G.; Zhang, X. H.; Li, Q. W. Appl. Phys. Lett. 2010, 97, 181906.

[36] Liu, W.; Zhang, X. H.; Xu, G.; Bradford, P. D.; Wang, X.; Zhao, H. B.; Zhang, Y. Y.; Jia, Q. X.; Yuan, F.; Li, Q. W.; Qiu, Y.; Zhu, Y. T. Carbon 2011, 49, 4786.

[37] Wang, X.; Bradford, P. D.; Liu, W.; Zhao, H. B.; Inoue, Y.; Maria, J.; Li, Q. W.; Yuan, F.; Zhu, Y. T. Compos. Sci. Technol. 2011, 71, 1677.

[38] Foroughi, J.; Spinks, G. M.; Ghorbani, S. R.; Kozlov, M. E.; Safaei, F.; Peleckis, G.; Wallace, G. G.; Baughman, R. H. Nanoscale 2012, 4, 940.

[39] Jiang, K. L.; Li, Q. Q.; Fan, S. S. Nature 2002, 419, 801.

[40] Zhang, M.; Atkinson, K. R.; Baughmen, R. H. Science 2004, 306, 1358.

[41] Zhang, M.; Fang, S.; Zahidov, A. A.; Lee, S. B.; Aliev, A. E.; Williams, C. D.; Atkinson, K. R.; Baughman, R. H. Science 2005, 309,1215

[42] Li, Q. W.; Zhang, X. F.; DePaula, R. F.; Zheng, L. X.; Zhao, Y.; Stan, L.; Holesinger, T. G.; Arendt, P. N.; Reterson, D. E.; Zhu, Y. T. $A d v$. Mater. 2006, 18, 3160.

[43] Inoue, Y.; Kakihata, K.; Hirono, Y.; Horie, T.; Ishida, A.; Minura, H. Appl. Phys. Lett. 2008, 92, 213113.

[44] Zhang, S.; Zhu, L.; Minus, M.; Chae, H.; Jagannathan, S.; Wong, C.; Kowalik, J.; Roberson, L. B.; Kumar, S. J. Mater. Sci. 2008, 43, 4356.

[45] Nakayama, Y. J. Jpn. J. Appl. Phys. 2008, 47, 8149.

[46] Zhang, Q.; Wang, D. G.; Huang, J. Q.; Zhou, W. P.; Luo, G. H.; Qian, W. Z.; Wei, F. Carbon 2010, 48, 2855.
[47] Huynh, C. P.; Hawkins, S. C. Carbon 2010, 48, 1105.

[48] Lee, I. H.; Han, G. H.; Chae, S. J.; Bae, J. J.; Kim, E. S.; Kim, S. M.; Kim, T. H.; Jeong, H. K.; Lee, Y. H. Nano 2010, 5, 31.

[49] Zheng, L. X.; Sun, G. Z.; Zhan, Z. Y. Small 2010, 6, 132.

[50] Zhang, X. B.; Jiang, K. L.; Feng, C.; Liu, P.; Zhang, L.; Kong, J.; Zhang, T.; Li, Q. Q.; Fan, S. S. Adv. Mater. 2006, 18, 1505.

[51] Liu, K.; Sun, Y. H.; Chen, L.; Feng, C.; Feng, X. F.; Jiang, K. L.; Zhao, Y. G.; Fan, S. S. Nano Lett. 2008, 8, 700.

[52] Kuznetsov, A. A.; Fonseca, A. F.; Baughman, R. H.; Zakhidov, A. A. ACS Nano 2011, 5, 985.

[53] Gilvaei, A. F.; Hirahara, K.; Nakayama, Y. Carbon 2011, 49, 4928.

[54] Jiang, K. L.; Wang, J. P.; Li, Q. Q.; Liu, L.; Liu, C. H.; Fan, S. S. Scientia Sinica Phys. Mech. \& Astron. 2011, 41, 390. (姜开利, 王佳 平, 李群庆, 刘亮, 刘长洪, 范守善, 中国科学: 物理学 力学 天文学, 2011, 41, 390.)

[55] Kim, J. H.; Jang, H. S.; Lee, K. H.; Overzet, L. J.; Lee, G. S. Carbon 2010, 48, 538.

[56] Choi, B. H.; Yoo, H.; Kim, Y. B.; Lee, J. H. Microelectron. Eng. 2010, 87,1500 .

[57] Nessim, G. D.; Hart, A. J.; Kim, J. S.; Acquaviva, D.; Oh, J.; Morgan, C. D.; Seita, M.; Leib, J. S.; Thompson, C. V. Nano Lett. 2008, $8,3587$.

[58] Zhang, Y. Y.; Zou, G.; Doorn, S. K.; Htoon, H.; Stan, L.; Hawley, M. E.; Sheehan, C. J.; Zhu, Y. T.; Jia, Q. X. ACS Nano 2009, 3, 2157.

[59] Pidduck, A. J.; Haslam, S. D.; Bryan-Brown, G. P.; Bannister, R.; Kitely, I. D. Appl. Phys. Lett. 1997, 71, 2907.

[60] Stöhr, J.; Samant, M. G.; Cossy-Favre, A.; Díaz, J.; Momoi, Y.; Sdahara, S.; Nagata, T. Macromolecules 1998, 31, 1942.

[61] Zhang, X. F.; Li, Q. W.; Tu, Y.; Li, Y.; Coulter, J. Y.; Zheng, L. X.; Zhao, Y. H.; Jia, Q. X.; Peterson, D. E.; Zhu, Y. T. Small 2007, 3, 244.

[62] Zhang, X. F.; Li, Q. W.; Holesinger, T. G.; Arendt, P. N.; Huang, J. Y.; Kirven, P. D.; Clapp, T. G.; DePaula, R. F.; Liao, X. Z.; Zhao, Y. H.; Zheng, L. X.; Peterson, D. E.; Zhu, Y. T. Adv. Mater. 2007, 19, 4198.

[63] Tran, C. D.; Humphries, W.; Smith, S. M.; Huynh, C.; Lucas, S. Carbon 2009, 47, 2662.

[64] Beyerlein, I. J.; Porwal, P. K.; Zhu, Y. T.; Hu, K.; Xu, X. F. Nanotechnology 2009, 20, 485702.

[65] Zhang, X. H.; Li, Q. W. ACS Nano 2010, 4, 312.

[66] Fang, S. L.; Zhang, M.; Zakhidov, A. A.; Baughman, R. H. J. Phys.: Condens. Matter 2010, 22, 334221.

[67] Liu, K.; Sun, Y. H.; Zhou, R. F.; Zhu, H. Y.; Wang, J. P.; Liu, L.; Fan, S. S.; Jiang, K. L. Nanotechnology 2010, 21, 045708.

[68] Zhao, J. N.; Zhang, X. H.; Di, J. T.; Xu, G.; Yang, X. J.; Liu, X. Y.; Yong, Z. Z.; Chen, M. H.; Li, Q. W. Small 2010, 6, 2612.

[69] Jia, J. J.; Zhao, J. N.; Xu, G.; Di, J. T.; Yong, Z. Z.; Tao, Y. Y.; Fang, C.; Zhang, Z. G.; Zhang, X. H.; Zheng, L. X.; Li, Q. W. Carbon 2011, 49, 1333.

[70] Miao, M. H.; Hawkins, S. C.; Cai, J. Y.; Gengenbach, T. R.; Knott, R.; Huynh, C. P. Carbon 2011, 49, 4940.

[71] Yang, Z. B.; Sun, X. M.; Chen, X. L.; Yong, Z. Z.; Xu, G.; He, R. X.; An, Z. H.; Li, Q. W.; Peng, H. S. J. Mater. Chem. 2011, $21,13772$.

[72] Tran, C.-D.; Lucas, S.; Phillips, D. G.; Randeniya, L. K.; Baughman, R. H.; Tran-Cong, T. Nanotechnology 2011, 22, 145302.

[73] Ryu, S.; Lee, Y.; Hwang, J.-W.; Hong, S.; Kim, C.; Park, T. G.; Lee, H.; Hong, S. H. Adv. Mater. 2011, 23, 1971.

[74] Deng, F.; Lu, W.; Zhao, H.; Zhu, Y. T.; Kim, B.-S.; Chou, T.-W. Carbon 2011, 49, 1752.

[75] Zu, M.; Li, Q. W.; Zhu, Y. T.; Dey, M.; Wang, G. J.; Lu, W. B.; Deitzel, J. M.; Gillespie Jr., J. W.; Byun, J.-H.; Chou, T.-W. Carbon 2012, 50, 1271 . 